\title{
Rosuvastatin Calcium
}

National Cancer Institute

\section{Source}

National Cancer Institute. Rosuvastatin Calcium. NCI Thesaurus. Code C61933.

The calcium salt form of rosuvastatin, a statin with antilipidemic activity. Rosuvastatin selectively and competitively binds to and inhibits hepatic hydroxymethyl-glutaryl coenzyme A (HMG-CoA) reductase, the enzyme which catalyzes the conversion of HMGCoA to mevalonate, a precursor of cholesterol. This leads to a decrease in hepatic cholesterol levels and increase in uptake of LDL cholesterol. 